\title{
A nonlinear elasto-plastic analysis of Reissner-Mindlin plates by finite element method
}

\author{
Meftah Kamel \\ University of Biskra, Laboratoire de Génie Energétique et Matériaux, LGEM, Faculty of Sciences and Tecbnology, Biskera, \\ 07000, Algeria \\ k.meftah@univ-biskra.dz, bttp://orcid.org/0000-0002-5671-602X
}

Sedira Lakhdar

University of Biskra, Laboratoire de Génie Mécanique, LGM, Faculty of Sciences and Technology, Biskra, 07000, Algeria

l.sedira@univ-biskra.dz, bttp://orcid.org/0000-0003-1735-2195

\begin{abstract}
In this paper, a finite element simulation of nonlinear elastoplastic deformations of Reissner-Mindlin bending plates is described. The previously proposed four-node Q4 $\gamma$ element with transverse energy of shearing for thick bending plates is extended to account for isotropic material nonlinearities. An incremental finite element procedure has been used for the elasto-plastic analysis of the thick bending plate. Modified Newton-Raphson method has been used to solve the nonlinear equations. Von-Mises yield criteria have been applied for yielding of the materials along with the associated flow rule. To verify the present element, simple tests are demonstrated and various elasto-plastic problems in which the development of the plastic zone are solved.
\end{abstract}

KEYwORDS. Plate element; Reissner-Mindlin plates; Elasto-plasticity; Nonlinear analysis.

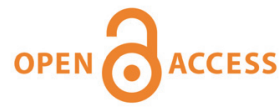

Citation: Meftah, K., Sedira, L., A nonlinear elasto-plastic analysis of Reissner-Mindlin plates by finite element method, Frattura ed Integrità Strutturale, 50 (2019) 276-285.

Received: 26.06 .2019

Accepted: 16.08 .2019

Published: 01.10.2019

Copyright: (C) 2019 This is an open access article under the terms of the CC-BY 4.0, which permits unrestricted use, distribution, and reproduction in any medium, provided the original author and source are credited.

\section{INTRODUCTION}

I $\mathrm{n}$ recent years, considerable work has been devoted to the study of nonlinear elasto-plastic responses of ReissnerMindlin bending plates, since plates are very important parts of engineering structures. The nonlinear elasto-plastic bending plates are analyzed by the finite element methods, the finite difference methods, the discrete element methods and the direct numerical methods. However, the finite element method is a suitable approach and has been successfully used in nonlinear elasto-plastic analysis of plates. For example, a finite element analysis of Reissner-Mindlin bending plates has been investigated by Owen and Hinton [1], Meftah [2], Rezaiee-Pajand and Sadeghi [3] and Kanber and Bozkurt [4]. The finite volume formulation is also adopted for the elasto-plastic analysis of Reissner-Mindlin plates by adapting the layered approach [5] and non-layered model [6]. The elasto-plastic analysis using the Element Free Galerkin 
method (EFGM) was initially applied to fracture problems and subsequently applied to 2D problems and to 3D problems $[7,8]$. On the other hand, the incremental cyclic plasticity theory is recently used firstly to determine the actual stress and strain state arising in two-dimensional or axi-symmetric notched components and later is extended by Marangon et al. [9] and Campagnolo et al. [10] to study the three-dimensional effects at the tip of rounded notches in plates of finite thickness.

The first order shear deformation theories (FSDTs), which include transverse shear deformation, for bending plates have been initially proposed by Reissner [11] and further developed by Mindlin [12]. These theories are widely employed in the nonlinear elasto-plastic behavior.

In this study, a finite element method for analyzing the elasto-plastic plate bending problems is presented. The previous Q4 $\gamma$ [13] plate element with transverse energy of shearing is extended to account for nonlinear elasto-plastic. The goal of this work is to present a transverse energy four-node element Q4 $\gamma$ with only four corner nodes which is significantly superior to the classical four-node element and is not computationally as expensive as a quadratic quadrangle 9-noded Hétérosis element. Having a finite element method for linear elastic analysis of Reissner-Mindlin bending plates, we further develop the model to investigate the elasto-plastic behavior and plastic zone of the structures under consideration. A modified Newton-Raphson method has been used to solve the non-linear equations. Von-Mises yield criterion has been adopted to deal with yielding of the materials along with the isotropic hardening. A computer program has been developed and a number of plate-bending problems have been solved. As the applications of the present element, the square plates with the various boundary conditions are calculated. The results have been compared with existing benchmark solutions. Results obtained with the Q4 $\gamma$ plate element, with the adopted constitutive laws, are compared with those provided by the quadratic quadrangle 9-noded Hétérosis element presented in References [1,14] with the provision of selective integration and reduced integration. All the computations were carried out in FORTRAN Finite Element code developed by Owen and Hinton [1] and Hinton and Owen [14].

\section{REISSNER-MINDLIN PLATE THEORY}

7 he Reissner-Mindlin plate theory (also designated first order shear deformation theory, FSDT) is more adequate for the analysis of moderately thick plates. The sign convention for stress resultants, the displacement field and the coordinate system are indicated in Fig. 1. General notation is $M_{x}, M_{y}$ bending moments, $M_{x y}$ twisting moments, $V_{x}, V_{y}$ transverse shear forces, $w$ deflection in $z$-direction and $\theta_{x}, \theta_{y}$ rotations of the $x z_{-}$and $y z$-planes, respectively.

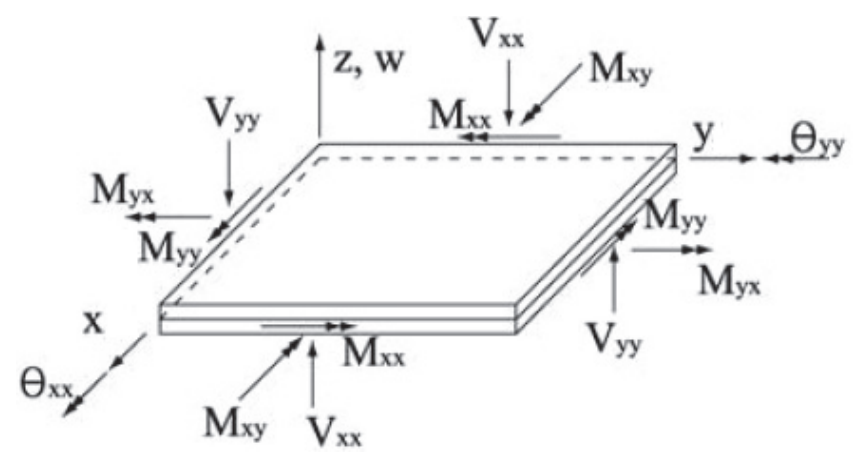

Figure 1: Schematic of the Reissner-Mindlin plate indicating the sign convention chosen for forces and moments.

The displacement field at any point within the element is given by:

$$
\left\{\begin{array}{c}
u=-z \theta_{x}(x, y) \\
v=-z \theta_{y}(x, y) \\
w=w(x, y)
\end{array}\right.
$$

The flexural and transverse shear strains in the plate for isotropic homogeneous linear behavior elastic can be written in the concise matrix form as: 


$$
\left\{\varepsilon_{f}\right\}=\left\{\begin{array}{lll}
\varepsilon_{x} & \varepsilon_{y} & \gamma_{x y}
\end{array}\right\}^{T} ;\left\{\varepsilon_{s}\right\}=\left\{\begin{array}{ll}
\gamma_{x z} & \gamma_{y z}
\end{array}\right\}^{T}
$$

The linear relationships between the displacements and strains can be obtained by using the definitions of strains from the theory of elasticity:

$$
\begin{aligned}
& \varepsilon_{x}=\frac{\partial u}{\partial x}=-z \frac{\partial \theta_{x}}{\partial x} ; \quad \varepsilon_{y}=\frac{\partial v}{\partial y}=-z \frac{\partial \theta_{y}}{\partial y} \\
& \gamma_{x y}=2 \varepsilon_{x y}=\frac{\partial u}{\partial y}+\frac{\partial v}{\partial x}=-z\left(\frac{\partial \theta_{x}}{\partial y}+\frac{\partial \theta_{y}}{\partial x}\right) \\
& \gamma_{x z}=2 \varepsilon_{x z}=\frac{\partial w}{\partial x}-\theta_{x} ; \gamma_{y z}=2 \varepsilon_{y z}=\frac{\partial w}{\partial y}-\theta_{y}
\end{aligned}
$$

Assuming normal stress $\sigma_{\text {z: }}$ to be negligibly small compared to other normal stresses, the stress-strain relationship in the matrix takes the form:

$$
\sigma=[D] \varepsilon
$$

where $\{\sigma\}=\left\{\sigma_{x x} \sigma_{y y} \tau_{x y} \tau_{y z} \tau_{x x}\right\}^{\mathrm{T}}$ and the matrix $[D]$ for isotropic materials is defined:

$$
[D]=\left[\begin{array}{cc}
D_{f} & 0 \\
0 & D_{s}
\end{array}\right]
$$

with

$$
\left[D_{s}\right]=G . k . h\left[\begin{array}{cc}
1 & 0 \\
0 & 1
\end{array}\right] ;\left[D_{f}\right]=\frac{E \cdot h^{3}}{12\left(1-v^{2}\right)}\left[\begin{array}{ccc}
1 & v & 0 \\
v & 1 & 0 \\
0 & 0 & \frac{1-v}{2}
\end{array}\right]
$$

where $b$ is the thickness of the plate, $k$ is a shear correction coefficient, $E$ is the Young's modulus and $v$ is the Poisson's ratio.

The generalized forces per unit of length of the plate side can be obtained using the stress field; these forces are the bending moments $(M)$ and the shear forces $(V)$ :

$$
\{M\}=\int_{-\frac{h}{2}}^{\frac{b}{2}}-z\left\{\begin{array}{c}
\sigma_{x x} \\
\sigma_{y y} \\
\tau_{x y}
\end{array}\right\} d z ;\{V\}=\int_{-\frac{b}{2}}^{\frac{b}{2}}\left\{\begin{array}{c}
\tau_{y z} \\
\tau_{x z}
\end{array}\right\} d z
$$

\section{CONSTITUTIVE EQUATION FOR RATE INDEPENDENT ELASTOPLASTICITY}

A fter initial yielding the material behavior will be partly elastic and partly plastic. During any increment of stress, the changes of strain are assumed to be divisible into elastic and plastic components, so that: 


$$
d \varepsilon=d \varepsilon_{e}+d \varepsilon_{p}
$$

The elastic-plastic strain increment is given by the incremental form of:

$$
d \varepsilon=[D]^{-1} d \sigma+d \lambda \frac{\partial F}{\partial \sigma}
$$

where $d \lambda$ is a proportional constant called plastic multiplier and $\frac{\partial F}{\partial \sigma}$ is the flow vector, which is normal to the adopted yield function, presented in Eq. (12), considering that the yield surface only depends on the magnitude of the applied principal stresses and of a hardening parameter $H$ :

$$
F(\sigma, H)=f(\sigma, H)-\sigma_{Y}(H)=0
$$

where $\sigma_{Y}$ is the yield stress and $f(\sigma, H)$ is the yield criterion. In this paper, the elasto-plastic constitutive model based on the Von-Mises associated yield criterion is adopted for the Reissner-Mindlin plate theory [15]:

$$
f(\sigma)=\bar{\sigma}=\frac{1}{\sqrt{2}}\left[\left(\sigma_{x x}+\sigma_{y y}\right)^{2}+\sigma_{x x}^{2}+\sigma_{y y}^{2}+6\left(\tau_{x y}^{2}+\tau_{y z}^{2}+\tau_{x x}^{2}\right)\right]^{\frac{1}{2}} \leq \sigma_{Y}
$$

where $\sigma_{e}$ is the Von-Mises effective stress.

The elastic-plastic incremental stress strain relationship:

$$
\begin{aligned}
& d \sigma=\left[D^{e p}\right]\left(d \varepsilon_{e}+d \varepsilon_{p}\right) \\
& d \sigma=\left[D^{e p}\right]\left(d \varepsilon_{e}+d \lambda \frac{\partial F}{\partial \sigma}\right)
\end{aligned}
$$

The differential form of Eq. (12) is:

$$
d \mathrm{~F}=\frac{\partial f}{\partial \sigma} d \sigma-\frac{\partial \sigma_{Y}}{\partial H} H=0
$$

or

$$
d F=\mathrm{a}^{\mathrm{T}} d \sigma-A d \lambda=0
$$

in which the flow vector $\mathrm{a}^{\mathrm{T}}$ is define as:

$$
\mathrm{a}^{\mathrm{T}}=\frac{\partial f}{\partial \sigma}=\left[\begin{array}{lllll}
\frac{\partial f}{\partial \sigma_{x}} & \frac{\partial f}{\partial \sigma_{y}} & \frac{\partial f}{\partial \tau_{x y}} & \frac{\partial f}{\partial \tau_{y z}} & \frac{\partial f}{\partial \tau_{z x}}
\end{array}\right]
$$

Eqns. (16) and (17) can be reduced to get the hardening parameter $A$ as:

$$
A=d \lambda^{-1} \frac{\partial \sigma_{Y}}{\partial H} H
$$

The plastic rate multiplier can be obtained as: 


$$
d \lambda=\frac{\mathrm{a}^{\mathrm{T}} D}{A+\mathrm{a}^{\mathrm{T}} D \mathrm{a}} d \varepsilon
$$

By substituting the expression of the plastic multiplier $d \lambda$ into Eq. (15), the elasto-plastic tangent modulus is derived as:

$$
D_{e p}=D-\frac{D \mathrm{a} \mathrm{a}^{\mathrm{T}} D}{A+\mathrm{a}^{\mathrm{T}} D \mathrm{a}}
$$

The incremental stress-strain relationship is given as:

$$
\left\{\begin{array}{l}
d \sigma_{f} \\
d \sigma_{s}
\end{array}\right\}=\left[\begin{array}{cc}
\left(D_{e p}\right)_{f} & 0 \\
0 & D_{s}
\end{array}\right]\left\{\begin{array}{l}
d \varepsilon_{f} \\
d \varepsilon_{s}
\end{array}\right\}
$$

For Mindlin plate, yield function $\mathrm{F}$ is assumed to be function of $\sigma_{f}$ but not of the transverse shear stresses $\sigma_{s}$, the direct stresses associated with flexure only hence $D_{s}$ always remain elastic $[1,16,17]$.

\section{FINITE ELEMENT FORMULATION}

he Mindlin-Reissner theory takes the shear deformation into account by decoupling the rotation of the plate crosssection from the slope of the deformed mid-surface and the displacement field requires $\mathrm{C}^{0}$ continuity only. Then the displacement fields (the transverse displacement $w$ and two rotations $\theta_{x}, \theta_{y}$ ) are described by the same order of shape functions as follows:

$$
\{d\}=\left\{\begin{array}{l}
w \\
\theta_{x} \\
\theta_{y}
\end{array}\right\}=\sum_{i=1}^{n}\left[\begin{array}{ccc}
N_{i} & 0 & 0 \\
0 & N_{i} & 0 \\
0 & 0 & N_{i}
\end{array}\right]\left\{\begin{array}{l}
w_{i} \\
\theta_{x i} \\
\theta_{y i}
\end{array}\right\}
$$

The bending and shear strain-displacement relationships are given as:

$$
\delta \varepsilon_{f}=\sum_{i=1}^{n} B_{f i} \cdot \delta d_{i} ; \delta \varepsilon_{s}=\sum_{i=1}^{n} B_{s i} \cdot \delta d_{i}
$$

with

$$
B_{f i}=\left[\begin{array}{ccc}
0 & -\frac{\partial N_{i}}{\partial x} & 0 \\
0 & 0 & -\frac{\partial N_{i}}{\partial y} \\
0 & -\frac{\partial N_{i}}{\partial y} & -\frac{\partial N_{i}}{\partial x}
\end{array}\right] ; B_{s i}=\left[\begin{array}{ccc}
\frac{\partial N_{i}}{\partial x} & -N_{i} & 0 \\
\frac{\partial N_{i}}{\partial y} & 0 & -N_{i}
\end{array}\right]
$$

The tangential stiffness matrix can be written as follows:

$$
K_{T}=\int_{A}\left(\left[B_{f}\right]^{T}\left[D_{e p}\right]\left[B_{f}\right]+\left[B_{s}\right]^{T}[D]\left[B_{s}\right]\right) d A
$$


In this study the solution is obtained resorting to the modified Newton-Raphson method [1]. In this algorithm the modification consists of computing the tangent stiffness matrix only once in the beginning of each load increment than in each iteration.

\section{Q4 $\gamma$ finite element}

The Q4 $\gamma$ element shown in Fig. 2 is adopted in the present study. This element contains four nodes at the corners and the associated classical interpolation functions given by [13]:

$$
N_{i}=\frac{1}{4}\left(1+\xi \xi_{i}\right)\left(1+\eta \eta_{i}\right) ; i=1,2,3 \text { and } 4
$$

with $\left(\xi_{1}, \xi_{2}, \xi_{3}, \xi_{4}\right)=(-1,1,1,-1)$ and $\left(\eta_{1}, \eta_{2}, \eta_{3}, \eta_{4}\right)=(-1,-1,1,1)$.

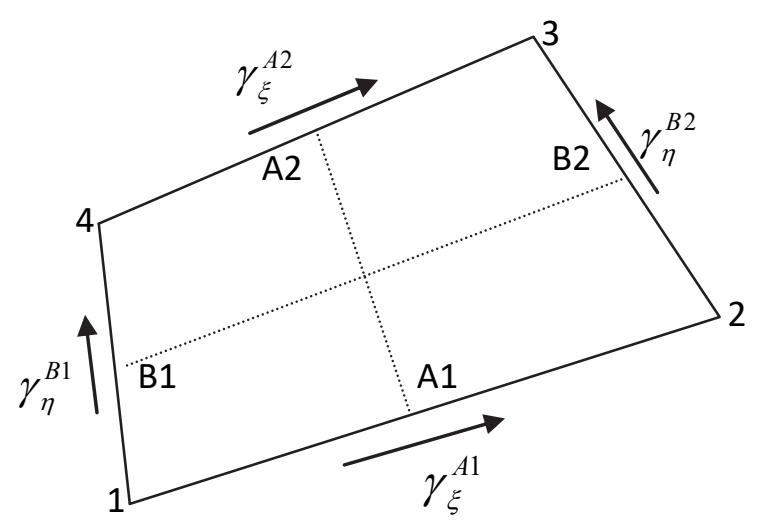

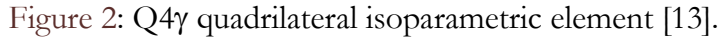

For the Q4 4 element the transverse field of distortion $\bar{\gamma}$ is linearly discretizes in the element of reference by side so that:

$$
\gamma=\left\{\begin{array}{l}
\bar{\gamma}_{\xi} \\
\bar{\gamma}_{\eta}
\end{array}\right\}=\left\{\begin{array}{l}
\frac{1-\eta}{2} \gamma_{\xi}^{A 1}+\frac{1+\eta}{2} \gamma_{\xi}^{A 2} \\
\frac{1-\xi}{2} \gamma_{\eta}^{B 1}+\frac{1+\xi}{2} \gamma_{\eta}^{B 2}
\end{array}\right\}
$$

By means of then the relations:

$$
\int_{-1}^{+1}\left(\bar{\gamma}_{\xi}-\left(w_{, \xi}+\theta_{\xi}\right)\right) d \xi=0 ; \int_{-1}^{+1}\left(\bar{\gamma}_{\eta}-\left(w_{, \eta}+\theta_{\eta}\right)\right) d \eta=0 ; \text { for } \xi= \pm 1 \text { and } \eta= \pm 1
$$

One establishes that:

$$
\begin{aligned}
& \gamma_{\xi}^{A 1}=\frac{1}{2}\left(w_{2}-w_{1}+\theta_{\xi_{1}}+\theta_{\xi_{2}}\right) ; \gamma_{\xi}^{A 2}=\frac{1}{2}\left(w_{4}-w_{3}+\theta_{\xi_{3}}+\theta_{\xi_{4}}\right) \\
& \gamma_{\xi}^{B 1}=\frac{1}{2}\left(w_{4}-w_{1}+\theta_{\xi_{1}}+\theta_{\xi_{4}}\right) ; \gamma_{\xi}^{B 2}=\frac{1}{2}\left(w_{3}-w_{2}+\theta_{\xi_{2}}+\theta_{\xi_{3}}\right)
\end{aligned}
$$

By deferring the two results above in the statement of $\bar{\gamma}$, one from of deduced that: 


$$
\gamma=\left\{\begin{array}{c}
\bar{\gamma}_{\xi} \\
\bar{\gamma}_{\eta}
\end{array}\right\}=\left[\begin{array}{ccc}
\frac{\partial N_{i}}{\partial \xi} & \xi_{i} \frac{\partial N_{i}}{\partial \xi} & 0 \\
\ldots N_{i} & 0 & \eta_{i} \frac{\partial N_{i}}{\partial \eta}
\end{array}\right]\left\{\begin{array}{c}
\vdots \\
w_{i} \\
\theta_{\xi_{i}} \\
\theta_{\eta i} \\
\vdots
\end{array}\right\}
$$

It is now necessary to express the rotations given here in the element of reference according to rotations in the local coordinate system:

$$
\left\{\begin{array}{c}
\theta_{\xi k} \\
\theta_{\eta k}
\end{array}\right\}=\left[J_{k}\right]\left\{\begin{array}{c}
\theta_{x k} \\
\theta_{y / k}
\end{array}\right\}
$$

where $J_{k}$ is the inverse Jacobian matrix components.

\section{NUMERICAL EXAMPLES}

he resulting mathematical model of the proposed Q4 $\gamma$ element and the classical associative plasticity model are implemented into a FORTRAN calculation code to account for small strain elasto-plastic problems. A nonlinear elasto-plastic behavior of bending plates under mechanical loading with different boundary conditions and different aspect ratios were studied. Both problems involve square plates subjected to a uniformly distributed load of magnitude $1 \mathrm{KN} / \mathrm{m}^{2}$. The material is considered as elasto-plastic (where the material is considered elastic perfectly plastic and the von Mises model is adopted) with: $L=1.0 ; h=0.5$ and $0.01 ; E=10.92 \mathrm{GPa} ; v=0.3$ and Yield stress $\sigma_{Y}=1600$ $\mathrm{MPa}$. Because of the symmetry, only a quarter of the plate is modeled using $4 \times 4$ mesh as shown in Fig. 3.

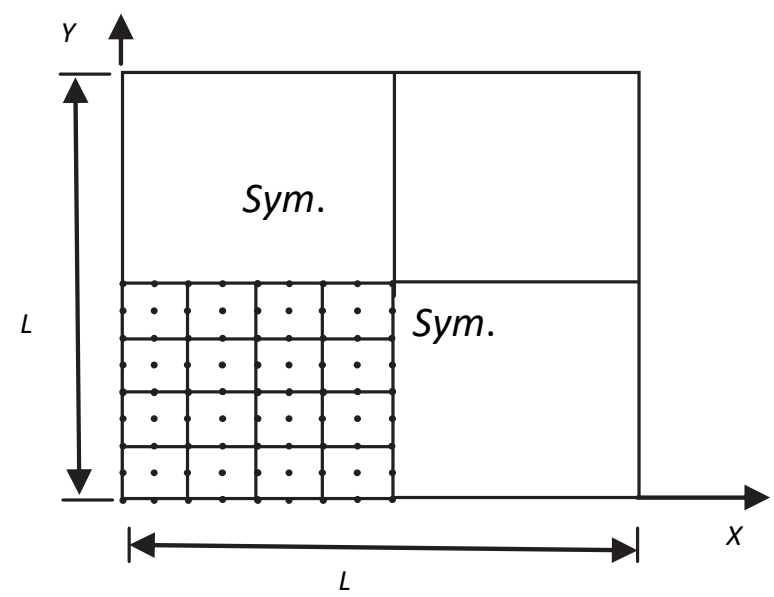

Figure 3: Square plate, its finite element models.

\section{Simply supported square plate}

In the first elasto-plastic example, a simply supported square plate subjected to uniformly distributed load is considered. The results are presented in Figs. 4 and 5 and shows the load-deflection curves with respect to the maximum deflection when nondimensional incremental load intensity is $q \cdot L^{2} / M_{0}\left(M_{0}\right.$ fully plastic moment $\left.M_{0}=\sigma_{Y} \cdot L \cdot b^{2} / 4\right)$. This figure also shows a comparison among the Hétérosis finite element solution obtained by Owen and Hinton [1]. The central displacements, for comparison of plates with different thicknesses ( $h=0.01$ and 0.5$)$, have been normalized as: $W . D / M_{0} \cdot L^{2}$ where $D=\left(E . h^{3}\right) /\left(12\left(1-v^{2}\right)\right)$ is the flexural rigidity of the plate. The results obtained from the present 4node element Q4 $\gamma$ agree well with those obtained from the 9-node Hétérosis element reported in Reference [1]. From the observation of the figures, it is possible to conclude that the accuracies of the present 4-node new element globally close 
to that of the 9-node Hétérosis element. Fig. 6 shows the progression of the plastic regions at different levels of loading. From this figure, first yielding is observed at the four corners of the plate and then at the center, and the plastic regions extend along the diagonals.

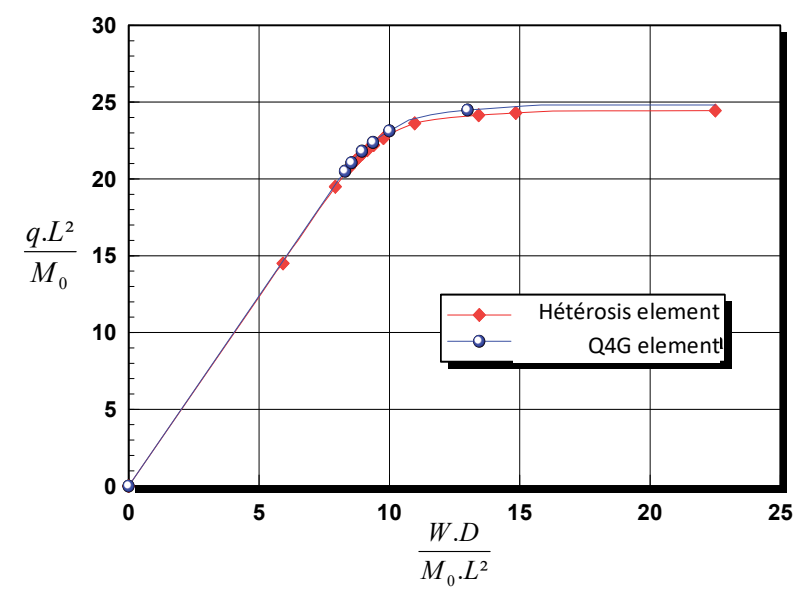

Figure 4: Load-deflection curves for simply supported square plate $(h=0.01)$.

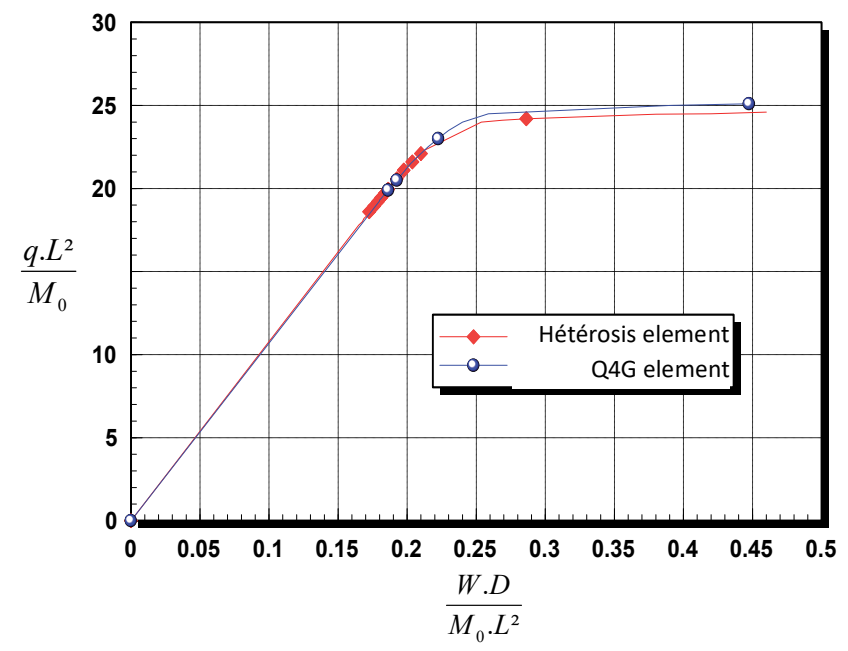

Figure 5: Load-deflection curves for simply supported square plate $(b=0.5)$.

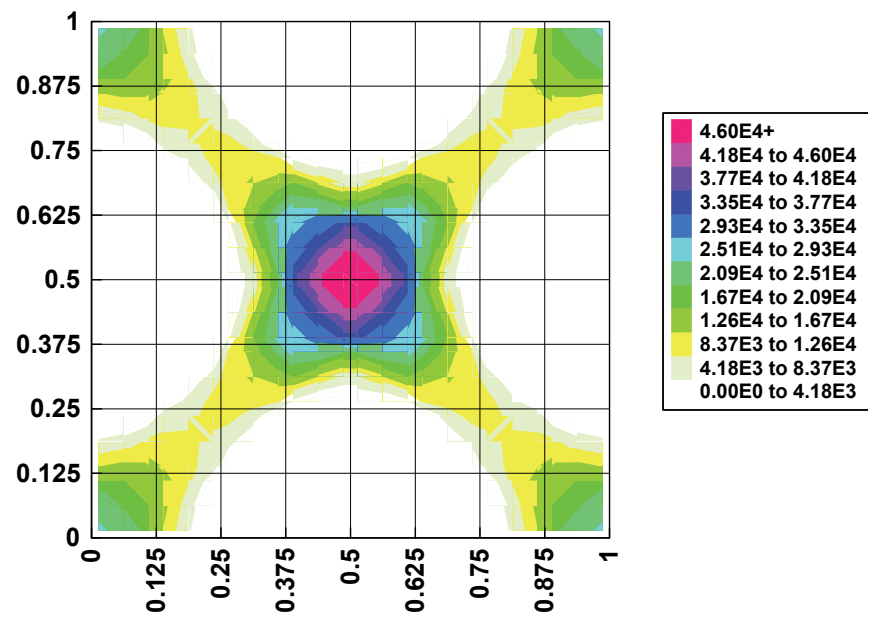

Figure 6: Progression of Yield regions for simply supported square plate $(b=0.01)$. 


\section{Clamped square plate}

A clamped square plate subjected to uniform load is analyzed. Fig. 7 shows the load-deflection curve with respect to maximum deflection with $(b=0.01)$. The present 4-node element $\mathrm{Q} 4 \gamma$ results are compared with those of Hétérosis finite element reported by Owen and Hinton [1]. From this figure, it is observed that the results provided by the present fournode Q4 $\gamma$ element are in a good agreement with those of the converged results of the 9-node Hétérosis element given in [1]. The progression of the yield regions at different levels of loading is summarized in Fig. 8. In this case, the first yielding of the plate occurs at the middle of the four edges, and the plastic regions extend along these edges until the center of the plate yields.

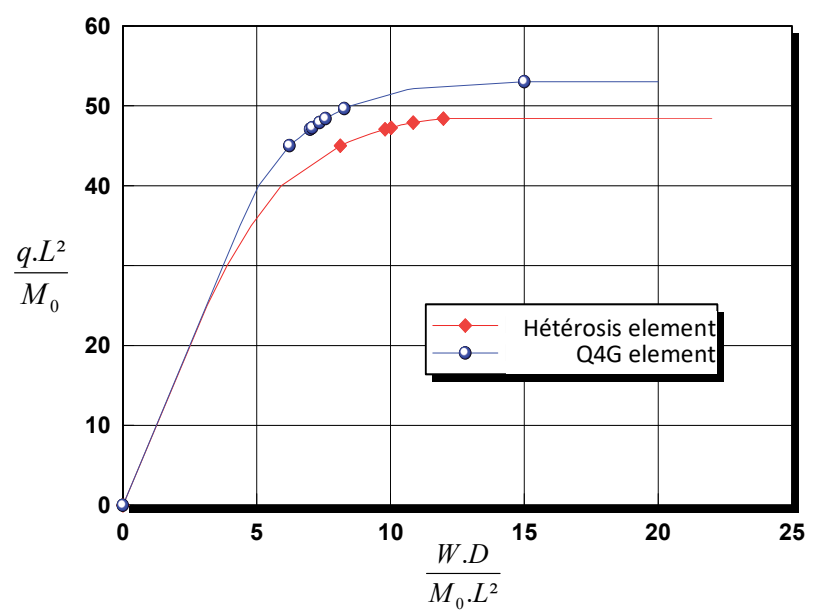

Figure 7: Load-deflection curves for clamped square plate $(b=0.01)$.

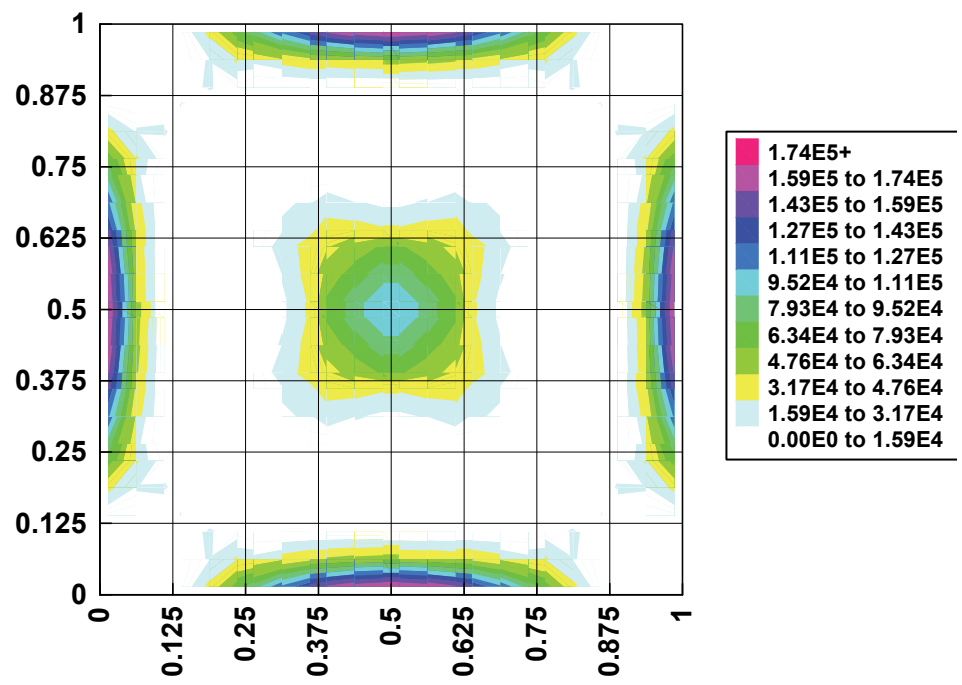

Figure 8: Progression of Yield regions for clamped square plate $(b=0.01)$.

\section{CONCLUSIONS}

$\mathrm{I}$ $\mathrm{n}$ this work, a finite element method for analyzing the problem of elasto-plastic bending of a square plate is presented. The previously proposed four-node Q4 $\gamma$ element with transverse energy of shearing for thick bending plates is extended to account for nonlinear elasto-plastic problems. The adopted constitutive model is the classical associative Von-Mises plasticity model where the modified Newton-Raphson scheme has been implemented for solving the nonlinear numerical system. To perform numerical tests, various examples within the nonlinear context are used to assess the accuracy against currently existing well-performed elements. The present element shows reliability and 
robustness when compared with some reference elements from the literature. The elasto-plastic results obtained by fournode Q4 $\gamma$ element for the square bending plates with various boundary conditions can be treated with acceptable accuracy compared with those obtained by 9-node Hétérosis element. For the displacement field and for the plastic zone the Q4 $\gamma$ solutions are very similar to the Hétérosis solutions.

\section{REFERENCES}

[1] Owen, D.R.J. and Hinton, E. (1980). Finite elements in plasticity - Theory and practice, Pinerdge Press Limited, Swansea, U.K.

[2] Meftah, K. (2019). Analyse non linéaire (élasto-plasticité) des plaques Reissner-Mindlin, Ed. Universitaires Europeennes, Paris, France.

[3] Rezaiee-Pajand, M. and Sadeghi, Y. (2013). A bending element for isotropic, multilayered and piezoelectric plates, Latin American Journal of Solids and Structures, 10(2), pp. 323-348. DOI: 10.1590/S1679-78252013000200006.

[4] Kanber, B. and Bozkurt, · O.Y. (2006). Finite element analysis of elasto-plastic plate bending problems using transition rectangular plate elements, Acta Mechanica Sinica 22, pp. 355-365. DOI: 10.1007/s10409-006-0012-y.

[5] Fallah, N. and Parayandeh-Shahrestany A. (2014). A novel finite volume based formulation for the elasto-plastic analysis of plates, Thin-Walled Structures, 77, pp. 153-164. DOI: 10.1016/j.tws.2013.09.025.

[6] Fallah, N., Parayandeh Shahrestany, A. and Golkoubi, H. (2017). A Finite Volume Formulation for the Elasto-Plastic Analysis of Rectangular Mindlin-Reissner Plates, a Non-Layered Approach, Civil Engineering Infrastructures Journal, 50(2), pp. 293 - 310. DOI: 10.7508/CEIJ.2017.02.006.

[7] Kargarnovin, M.H., Toussi, H.E. and Fariborz, S.J. (2003). Elasto-plastic element-free Galerkin method, Computational Mechanics, 33, pp. 206-14. DOI: 10.1007/s00466-003-0521-5.

[8] Pamin, J., Askes, H. and Borst, R. (2003). Two gradient plasticity theories discretized with the element-free Galerkin method, Computer Methods in Applied Mechanics and Engineering, 192, pp. 2377-403. DOI: $10.1007 /$ s00466-003-0521-5.

[9] Marangon, C., Campagnolo, A. and Berto, F. (2015). Three-dimensional effects at the tip of rounded notches subjected to mode-I loading under cyclic plasticity, J. Strain Anal. Eng. Des., 50(5), pp. 299-313. DOI: $10.1177 / 0309324715581964$.

[10] Campagnolo, A., Berto, F. and Marangon, C. (2016). Cyclic plasticity in three-dimensional notched components under in-phase multiaxial loading at $\mathrm{R}=-1$, Theor. Appl. Fract. Mech., 81. DOI: 10.1016/j.tafmec.2015.10.004.

[11] Reissner, E. (1945). The effect of transverse shear deformation on the bending of elastic plates. J. Appl. Mech., 12, pp. 66-77.

[12] Mindlin, R.D. (1951). Influence of rotatory inertia and shear on flexural motion of isotropic, elastic plates, J. Appl. Mech., 18, pp. 31-38.

[13] Batoz, J. and Dhatt, G. (1992). Modelization of structures by finite elements: beams and plates, Hermes, Paris.

[14] Hinton, E. and Owen, D.R.J. (1984). Finite element software for plates and shells, Pineridge Press Limited, Swansea, U.K.

[15] Chen, W.F. and Han, D.J. (1988). Plasticity for structural engineers, Springer-Verlag, New York.

[16] Kanber, B. and Bozkurt, O.Y. (2006). Finite element analysis of elasto-plastic plate bending problems using transition rectangular plate elements, Acta Mechanica Sinica, 22, pp. 355-365. DOI: 10.1007/s10409-006-0012-y.

[17] Belinha, J. and Dinis, L. (2006). Elasto-plastic analysis of plates by the element free Galerkin method, International Journal for Computer-Aided Engineering and Software, 23, pp. 525-551. DOI: 10.1108/02644400610671126. 